\title{
Entropy Production and Equilibrium Conditions in General-Covariant Continuum Physics
}

\author{
W. Muschik* and H.-H. von Borzeszkowski ${ }^{\dagger}$ \\ Institut für Theoretische Physik \\ Technische Universität Berlin \\ Hardenbergstr. 36 \\ D-10623 BERLIN, Germany
}

\begin{abstract}
Starting out with an entropy identity, the entropy flux, the entropy production and the corresponding Gibbs and Gibbs-Duhem equations of general-covariant continuum thermodynamics are established. Non-dissipative materials and equilibria are investigated. It is proved that equilibrium conditions only put on material properties cannot generate equilibria, rather additionally, the Killing property of the 4-temperature is a necessary condition for space-times in which equilibria are possible.
\end{abstract}

\section{Introduction}

The special-relativistic version of Continuum Thermodynamics (CT) was founded by Eckart [1] in form of the special-relativistic theory of irreversible processes. CT is based (i) on the conservation laws for the particle number and the energy-momentum tensor and (ii) on the dissipation inequality and the Gibbs fundamental law. In order to incorporate CT in (or, at least, to harmonize it with) General Relativity, as a first step, one has to formulate it on a curved space-time, i.e., to go over to its general-covariant formulation. This step brings problems along that one has to solve before taking Einstein's gravitational field equations into consideration. This paper is devoted to some of these problems. In particular, it concerns questions as to the entropy production, the Gibbs equation and the definition of thermodynamic equilibrium.

As to thermodynamic equilibrium, vanishing entropy production is a necessary condition to be satisfied. As is well-known [2, 3], this can be reached by two different requirements: Either by assuming that the considered matter is a perfect fluid (then one need not impose any conditions on the properties of the underlying space-time

${ }^{*}$ Corresponding author: muschik@physik.tu-berlin.de

†borzeszk@mailbox.tu-berlin.de 
structure) or by requiring space-time structures that allow for a Killing and a conform Killing vector field, respectively (then one need not restrict the structure of the material).

To define thermodynamic equilibrium, in both cases, the condition of vanishing entropy production has to be supplemented by further equilibrium conditions. It should be mentioned that for the second case it was shown 3 that, if the temperature 4-vector is a Killing vector, shear and expansion of the material vanish, and furthermore, that by implying Einstein's gravitational field equations, one can deduce most of the other supplementary conditions defining an equilibrium. In the present paper, the Killing equation and Einstein's equations are not exploited.

In the following, first we start out with an identity for the entropy 4-vector [4, 5]. This identity is split into the entropy production, the entropy flux and the Gibbs equation which is connected with the entropy density by a Gibbs-Duhem equation. As usual, the Gibbs equation allows the definition of a state space which is here one of local equilibrium because of the special choice of the entropy identity.

Different forms of the entropy production are considered for discussing non-dissipative materials and equilibria. Non-dissipative materials characterized by materialindependent vanishing of entropy production are rediscovered as perfect fluids [2]. For defining equilibrium, beyond vanishing entropy production additionally "supplementary equilibrium conditions" are required [5] and are introduced ad hoc. A general result is that in equilibrium the Killing property of the 4-temperature is valid for all materials.

\section{Energy-momentum Tensor}

The energy-momentum tensor $\Theta^{a b}$ is the governing field of GCCT11. Its (3+1)-split is

$$
\begin{aligned}
& \Theta^{k l}=\frac{1}{c^{4}} e u^{k} u^{l}+\frac{1}{c^{2}} u^{k} q^{l}+\frac{1}{c^{2}} q^{k} u^{l}+t^{k l}, \quad t^{k l}=t^{l k}, \\
& t^{k l}=-p h^{k l}+\pi^{k l}, \quad \pi^{k l} h_{k l}=0, \quad t^{k l} h_{k l}=t_{k}^{k}=:-3 p,
\end{aligned}
$$

with its components

$$
e=\Theta^{k l} u_{k} u_{l}, \quad q^{j}=\Theta^{k l} u_{l} h_{k}^{j}, \quad t^{i j}=\Theta^{k l} h_{k}^{i} h_{l}^{j} .
$$

Here, the projector perpendicular to the 4 -velocities $u^{k}$ and $u_{i}$ is

$$
h_{k}^{i}=\delta_{k}^{i}-\frac{1}{c^{2}} u^{i} u_{k}
$$

The meaning of the $(3+1)$-components is as follows: energy density $e$, energy flux density $q^{j}$, stress tensor $t^{i j}$, pressure $p$ and viscosity tensor $\pi^{k l}$.

\footnotetext{
${ }^{1}$ GCCT: General-Covariant $\underline{\text { Continuum }}$ Thermodynamics
} 


\section{Entropy Identity}

\subsection{The general case}

A continuum theory need beside the particle number balance which according to Eckart [1]2 reads

$$
N_{; k}^{k}=0, \quad N^{k}=\frac{1}{c^{2}} n u^{k}, \quad n:=N^{k} u_{k}, \quad\left(n u^{k}\right)_{; k}=0
$$

and the energy-momentum balance equation

$$
\Theta_{; k}^{k l}=0,
$$

an entropy balance

$$
S_{; k}^{k}=\sigma+\varphi, \quad S^{k}=\frac{1}{c^{2}} s u^{k}+s^{k}
$$

(particle flux density $N^{k}$, entropy 4 -vector $S^{k}$, entropy production $\sigma$, entropy supply $\varphi$ ). The Second Law of Thermodynamics is taken into account by the demand that the entropy production has to be non-negative at each event for arbitrary materials

$$
\sigma \geq 0
$$

For proceeding, we need the following

- Proposition [4, 5]: There exists an entropy identity

$$
S^{k} \equiv\left(s^{k}-\lambda q^{k}-\Lambda_{m} \Xi^{k m}\right)+\left(\mu N^{k}+\lambda u_{l} \Theta^{k l}+2 \Lambda_{p} h_{m}^{p} u_{n} S^{k n m}\right) .
$$

This identity contains quantities which stem from the $(3+1)$-splits of the energy-momentum tensor (11) 1 , of the entropy $(7)_{2}$, of the particle number flux density (5) 2 and from the (3+1)-split of the spin tensor

$$
S^{k a b}=\left(\frac{1}{c^{2}} s^{a b}+\frac{1}{c^{4}} u^{[a} \Xi^{b]}\right) u^{k}+s^{k a b}+\frac{1}{c^{2}} u^{[a} \Xi^{k b]} .
$$

The scalar $\mu$ is defined by

$$
\mu:=\frac{1}{n}\left(s-\lambda e-\Lambda_{m} \Xi^{m}\right)
$$

The two fields $\lambda$ and $\Lambda_{p}$ in (9) can be chosen arbitrarily: (9) is an identity, that means, it is valid for arbitrary $\lambda$ and $\Lambda_{p}$. According to (9) and (11), the part of $\Lambda_{m}$ which is parallel to $u_{m}$ does not contribute. The physical meaning of these two fields is determined by introducing below the general-relativistic Gibbs equation and the entropy flux which bring physics into the identity (9).

\footnotetext{
${ }^{2}$ We consider especially one-component systems.
} 


\subsection{Entropy identity of local equilibrium}

According to (7) 1 , we obtain the sum of entropy supply and production by calculating the divergence of (9). First of all the questions arises, how to distinguish between entropy production and entropy supply? In non-relativistic theories and here in thermodynamics on curved spaces 3 , this answer is easy to give: external forces and moments generates an entropy supply [5]. According to (6), here no external forces and moments are taken into account. Consequently, we have to cancel the entropy supply in (7) 1

$$
\varphi \equiv 0
$$

Because the energy-momentum tensor $\Theta^{k l}$ is spin-dependent and the spin appears explicitly in (9) by the terms which are multiplicated with $\Lambda_{m}$, the spin occurs twice in the entropy 4 -vector. This two-fold appearance of the spin can be specialized by the simplifying setting $\Lambda_{m} \doteq 0$ in the entropy identity (9). As we will see below, this setting generates a state space which belongs to local equilibrium. More general state spaces need a $\Lambda_{m}$ which is different from zero. The entropy identity (9) becomes in this special case

$$
S^{k} \equiv s^{k}-\lambda q^{k}+\frac{1}{n}(s-\lambda e) N^{k}+\lambda u_{l} \Theta^{k l} .
$$

According to (77) 1 and (12), the entropy identity (13) results in an other identity by differentiation

$$
\sigma=\left(s^{k}-\lambda q^{k}\right)_{; k}+\left[\frac{1}{n}(s-\lambda e) N^{k}\right]_{; k}+\left(\lambda u_{l} \Theta^{k l}\right)_{; k} .
$$

If the entropy flux density $s^{k}$ and the entropy density $s$ are specified in (14), this identity receives a physical meaning, if additionally the arbitrary field $\lambda$ is suitably chosen.

Using (7) $)_{2}$ and $(\underline{6})_{1}$, (14) results in

$$
\begin{aligned}
\sigma & =\left(s^{k}-\lambda q^{k}\right)_{; k}+\left(\frac{s}{n}\right)_{, k} N^{k}-\lambda\left(\frac{e}{n}\right)_{, k} N^{k}-\frac{e}{n} \lambda_{, k} N^{k}+ \\
& +\left(\lambda u_{m}\right)_{; k} \Theta^{k m} .
\end{aligned}
$$

Multiplying (11) by $u_{l}$

$$
u_{l} \Theta^{k l}=q^{k}+\frac{e}{n} N^{k}
$$

and taking (21) into account, the fifth term of (15) becomes

$$
\left(\lambda u_{m}\right)_{; k} \Theta^{k m}=\lambda_{, k}\left(q^{k}+\frac{e}{n} N^{k}\right)+\lambda u_{m ; k}\left(\frac{1}{c^{2}} u^{k} q^{m}-p h^{k m}+\pi^{k m}\right) .
$$

\footnotetext{
${ }^{3}$ without taking Einstein's equations into account
} 
Further, we have by use of (41) and (5) 4

$$
\lambda u_{m ; k} p h^{k m}=\lambda u_{; k}^{k} p=\lambda p n\left(\frac{1}{n}\right)^{\bullet},
$$

Finally, the identity (15) is according to (17), (18) and (15) 1 results in

$$
\begin{aligned}
\sigma & =\left(s^{k}-\lambda q^{k}\right)_{; k}+\left(\frac{s}{n}\right)_{, k} N^{k}-\lambda\left(\frac{e}{n}\right)_{, k} N^{k}-\lambda p n\left(\frac{1}{n}\right)^{\bullet}+ \\
& +\lambda_{, k} q^{k}+\lambda u_{m ; k}\left(\frac{1}{c^{2}} u^{k} q^{m}+\pi^{k m}\right)= \\
& =\left(s^{k}-\lambda q^{k}\right)_{; k}+\frac{n}{c^{2}}\left(\frac{s}{n}\right)^{\bullet}-\frac{n}{c^{2}} \lambda\left(\frac{e}{n}\right)^{\bullet}-\lambda p n\left(\frac{1}{n}\right)^{\bullet}+ \\
& +\lambda_{, k} q^{k}+\lambda u_{m ; k}\left(\frac{1}{c^{2}} u^{k} q^{m}+\pi^{k m}\right) .
\end{aligned}
$$

\section{Entropy Production and gr-Gibbs Equation}

As already mentioned, the identity (19) has to be transfered into the expression for the entropy production by specifying the entropy flux $s^{k}$ and the entropy density s. Obviously, (19) contains terms of different kinds: a divergence, time derivatives and two other terms. This fact gives rise to the following definitions of the entropy flux and the gr-Gibbs equation which generate the entropy production

$$
\begin{aligned}
s^{k} & :=\lambda q^{k} \longrightarrow \lambda=\frac{1}{T} \\
\left(\frac{s}{n}\right)^{\bullet} & :=\frac{1}{T}\left(\frac{e}{n}\right)^{\bullet}+c^{2} \frac{p}{T}\left(\frac{1}{n}\right)^{\bullet}, \\
\sigma & =\lambda_{, k} q^{k}+\lambda u_{m ; k}\left(\frac{1}{c^{2}} u^{k} q^{m}+\pi^{k m}\right) .
\end{aligned}
$$

The second term of (22) is

$$
\begin{aligned}
& \lambda u_{m ; k}\left(\frac{1}{c^{2}} u^{k} q^{m}+\pi^{k m}\right)=\lambda u_{m ; k}\left(\frac{1}{c^{2}} u^{k} q^{m}+\frac{1}{c^{2}} q^{k} u^{m}+\pi^{k m}\right)= \\
= & {\left[\left(\lambda u_{m}\right)_{; k}-\lambda_{, k} u_{m}\right]\left(\frac{1}{c^{2}} u^{k} q^{m}+\frac{1}{c^{2}} q^{k} u^{m}+\pi^{k m}\right)=} \\
= & \left(\lambda u_{(m)}\right)_{; k)}\left(\frac{1}{c^{2}} u^{k} q^{m}+\frac{1}{c^{2}} q^{k} u^{m}+\pi^{k m}\right)-\lambda_{, k} q^{k} .
\end{aligned}
$$

Consequently, the entropy production (22) takes another shape

$$
\sigma=\left(\lambda u_{(m}\right)_{; k)}\left(\frac{1}{c^{2}} u^{k} q^{m}+\frac{1}{c^{2}} q^{k} u^{m}+\pi^{k m}\right) .
$$


For the squel, wee need another expression containing the $(3+1)$-components of $\Theta^{k m}$ which do not appear in (24)

$$
\begin{array}{r}
\left(\lambda u_{m}\right)_{; k}\left(\frac{1}{c^{4}} e u^{k} u^{m}-p h^{k m}\right)=\left(\lambda_{, k} u_{m}+\lambda u_{m ; k}\right)\left(\frac{1}{c^{4}} e u^{k} u^{m}-p h^{k m}\right)= \\
=\lambda_{, k}\left(\frac{1}{c^{2}} e u^{k}\right)-\lambda u_{m ; k} p h^{k m}=\frac{e}{c^{2}} \lambda^{\bullet}-\lambda p u_{; k}^{k} .
\end{array}
$$

Summing up (24) and (25) results in another expression of the entropy production

$$
\sigma=\left(\lambda u_{(m}\right)_{; k)} \Theta^{k m}-\frac{e}{c^{2}} \lambda^{\bullet}+\lambda p u_{; k}^{k}
$$

We obtain a fourth form of the entropy production, if we decompose as usual [8] the velocity gradient into its kinematical invariants: symmetric traceless shear $\sigma_{n m}$, expansion $\Theta$, anti-symmetric rotation $\omega_{n m}$ and acceleration $\dot{u}_{n}$ :

$$
\begin{gathered}
u_{l ; k}=\sigma_{l k}+\omega_{l k}+\Theta h_{l k}+\frac{1}{c^{2}} \dot{u}_{l} u_{k} \\
\sigma_{l k}=\sigma_{k l}, \omega_{l k}=-\omega_{k l}, \quad u^{l} \sigma_{l k}=\sigma_{l k} u^{k}=u^{l} \omega_{l k}=\omega_{l k} u^{k}=0, \\
\sigma_{k}^{k}=\omega_{k}^{k}=0, \quad \Theta:=u_{k}^{k} .
\end{gathered}
$$

Consequently, the second term of (22) becomes with (22) 2 and (27)

$$
\lambda u_{l ; k}\left(\frac{1}{c^{2}} u^{k} q^{l}+\pi^{k l}\right)=\lambda\left(\frac{1}{c^{2}} \dot{u}_{l} q^{l}+\sigma_{l k} \pi^{k l}\right),
$$

and the entropy production (22) results in

$$
\sigma=\lambda_{, k} q^{k}+\lambda\left(\frac{1}{c^{2}} \dot{u}_{l} q^{l}+\sigma_{l k} \pi^{k l}\right)
$$

The question now arises, if the Pfaffian (21) is an integrable one ? To answer this question, we have to look for the entropy density belonging to the gr-Gibbs equation (21). We start out with an ansatz for $s$ and prove that it satisfies the gr-Gibbs equation (21), if a Gibbs-Duhem equation of the intensitiv variables is valid. Starting out with

$$
s:=\frac{e}{T}+c^{2} \frac{p}{T}
$$

and generating its differential

$$
\dot{s}=\frac{1}{T} \dot{e}+\left(e+c^{2} p\right)\left(\frac{1}{T}\right)^{\bullet}+\frac{c^{2}}{T} \dot{p}
$$

we demand as in thermostatics that the Gibbs-Duhem equation

$$
\left(e+c^{2} p\right)\left(\frac{1}{T}\right)^{\bullet}+\frac{c^{2}}{T} \dot{p}=0
$$


is valid for the intensitive variables. We obtain from (33) and (34)

$$
\frac{\dot{s}}{n}=\frac{1}{T} \frac{\dot{e}}{n}=\frac{1}{T}\left(\frac{e}{n}\right)^{\bullet}-\frac{e}{T}\left(\frac{1}{n}\right)^{\bullet}=\left(\frac{s}{n}\right)^{\bullet}-s\left(\frac{1}{n}\right)^{\bullet}
$$

This results in the gr-Gibbs equation (21) by use of (32)

$$
\left(\frac{s}{n}\right)^{\bullet}=\frac{1}{T}\left(\frac{e}{n}\right)^{\bullet}+\left(s-\frac{e}{T}\right)\left(\frac{1}{n}\right)^{\bullet}=\frac{1}{T}\left(\frac{e}{n}\right)^{\bullet}+c^{2} \frac{p}{T}\left(\frac{1}{n}\right)^{\bullet},
$$

that means, the entropy density (32) and the gr-Gibbs equation (21) are compatible with each other, if the Gibbs-Duhem equation (34) is introduced.

According to the gr-Gibbs equation (36), the state space is spanned by the energy per particle and the volume per particle

$$
\boxplus=\left(\frac{e}{n}, \frac{1}{n}\right) .
$$

This state space belongs to an one-component system in local equilibrium [6]. That is the reason why the identity (13) generated from (91) by seting $\Lambda_{m} \doteq 0$ was called the "entropy identity of local equilibrium". The constitutive quantities

$$
\mathbf{M}=\left(s / n, p, T, q^{k}, \pi^{k m}, \Xi^{k}, \Xi^{k m}\right)
$$

are functions of the state space variables

$$
\mathbf{M}=\mathcal{M}(\boxplus),
$$

which are called the constitutive equation 4 .

Special cases of space-times and materials are considered in the sequel.

\section{Non-dissipative Materials}

A non-dissipative material is characterized by vanishing entropy production even in the case of non-equilibrium 5 for all space-times. Consequently by definition, all processes in non-dissipative materials are reversible, and therefore these materials are those of thermostatics.

According to (22) and (24), the following material parameters are identical to zero for non-dissipative materials

$$
q_{\text {ndiss }}^{k} \equiv 0, \quad \pi_{\text {ndiss }}^{k l} \equiv 0 .
$$

According to (11) and (2), the energy-momentum tensor is that of a perfect fluid

$$
\Theta_{\text {ndiss }}^{k l}=\frac{1}{c^{4}} e u^{k} u^{l}-p h^{k l} .
$$

\footnotetext{
${ }^{4}$ How to use the constitutive equations in connection with the gravitational field equations see [7].

${ }^{5}$ Vanishing entropy production is necessary, but not sufficient for equilibrium.
} 
Consequently, we rediscover [2] the following

- Proposition: Non-dissipative materials are characterized by vanishing entropy production for arbitrary space-times, resulting in the material conditions (40): non-dissipative materials are perfect fluids.

The vanishing entropy production does not generate equilibria for non-dissipative materials according to (25) which represents an "equation of motion" of reversible processes. An other fact results from (25): vanishing entropy production is necessary for euilibrium but not sufficient for it, that means, we need beyond the vanishing entropy production additional equilibrium conditions which are considered in the next section.

\section{Equilibrium}

We start out with the question: How are equilibrium and non-dissipative materials related to each other ? Concerning non-dissipative materials, we are looking for material properties generating vanishing entropy production for arbitrary space-times independently of possible equilibria. Concerning equilibria, we are asking for those conditions which have to be satisfied by the space-time and by the actual material properties in equilibrium. Hereby, equilibrium is defined by equilibrium conditions which are divided into necessary and supplementary ones [5] which are idependent of each other The necessary ones are given by vanishing entropy production and vanishing entropy flux density

$$
\sigma^{e q} \doteq 0, \quad s_{e q}^{k} \doteq 0 .
$$

Independently, the supplementary equilibrium conditions are given by vanishing material time derivatives, except for that of the 4 -velocity

$$
\boxplus_{e q}^{\bullet} \doteq 0, \quad \boxplus \neq u^{l}
$$

From $(18)_{3}$ and (43) follows that the divergence of the 4-velocity vanishes in equilibrium for all materials

$$
u_{; k}^{k}{ }^{e q}=0 .
$$

The necessary equilibrium condition (42) 2 which stems from the entropy flux density becomes according to (20)

$$
q_{e q}^{k}=0 .
$$

The equilibrium condition (45) must not taken for (40) 1 which represent an invariable material property 6 , whereas (45) is only an actual one, valid in equilibrium for all materials. In non-equilibrium we have $q^{k} \neq 0$ which is not true for non-dissipative materials.. The equilibrium conditions (42) to (45)

\footnotetext{
${ }^{6}$ the heat conduction coefficients are zero
} 
are valid for all equilibria and all materials.

We now investigate equilibrium in more detail. From (22) follows with (42) 1 and (45)

$$
u_{m ; k}^{e q} \pi_{e q}^{k l}=0 \longrightarrow u_{(m ; k)}^{e q}=0 \vee \pi_{e q}^{k l}=0
$$

including (31)

$$
\sigma_{l k}^{e q} \pi_{e q}^{k l}=0 \quad \longrightarrow \quad \sigma_{l k}^{e q}=0 \vee \pi_{e q}^{k l}=0
$$

and (24) results in

$$
\left(\lambda u_{(m}\right)_{; k)}^{e q}=0 \vee \pi_{e q}^{k l}=0 .
$$

According to (42) $)_{1}$, (43) and (44), we obtain a sufficient and necessary equilibriun condition from (26) for arbitrary materials

$$
0=\left(\lambda u_{(m}\right)_{; k)}^{e q} \Theta_{e q}^{k m}=\left[\left(\frac{u_{m}}{T}\right)_{; k}^{e q}+\left(\frac{u_{k}}{T}\right)_{; m}^{e q}\right] \Theta_{e q}^{k m} .
$$

Because the energy-momentum tensor $\Theta^{k m}$ does not contain the temperature, we obtain the Killing condition for the 4-temperature $\lambda u_{m}$ as a general equilibrium condition

$$
0=\left[\left(\lambda u_{m}\right)_{; k}+\left(\lambda u_{k}\right)_{; m}\right]^{e q} \text {. }
$$

An interesting result is: Material properties subjected to equilibrium conditions cannot generate equilibria. Additionally, the Killing property (50) is necessary characterizing the space-times which allows equilibria.

Finally, a remark on an expression which can be found in literature [2, 8] may be useful: Subtracting (25) from (26) results in

$$
\sigma=\left(\lambda u_{(m)}\right)_{; k)}\left[\Theta^{k m}-\left(\frac{1}{c^{4}} u^{k} u^{m}-p h^{k m}\right)\right] .
$$

Here, $\sigma=0$ is not sufficient for equilibrium, because $\lambda^{\bullet}$ and $u_{; k}^{k}$ can be different from zero in (25) and (26). If equilibrium is presupposed7, the material behaves as a perfect fluid independently of the space-time, or if not, the spacetime has to obey (50) $)^{8}$. This is in accordance with the statement: $\sigma=0$ is necessary, but not sufficient for equilibrium.

\section{Discussion}

Usually, relativistic thermodynamics starts out with a symmetric and divergence-free energy-momentum tensor and an ansatz for the entropy 4-vector [8]

\footnotetext{
${ }^{7}$ that means, $\lambda^{\bullet}$ and $u^{k} ; k$ are zero in (25) and (26)

${ }^{8}$ or the 4 -temperature has to be a conform-Killing vector [3]
} 
whose divergence allows to find out the entropy production. The shortcoming of such a procedure is that the initial ansatz contains no hint at determining also the entropy flux fitting to that chosen entropy production. That is here the reason for starting out with an always valid entropy identity [4, 5] which allows to be split into three parts -entropy flux and production and Gibbs equation- which fit together. Here, a restricted entropy identity is taken up, because only systems in local equilibrium are investigated. Different forms of the entropy production are obtained, emphazising energy transport and viscosity or shear, or showing off the temperature 4-vector. Gibbs equation for entropy per particle number can be defined unambiguously. The integrability of this Gibbs equation is confirmed by a Gibbs-Duhem equality.

Non-dissipative materials are defined by vanishing entropy production independently of the actual space-time: perfect fluids are rediscovered as the only non-dissipative material [2]. Although the entropy production is identical to zero for all perfect fluids, there exist non-equilibria for them: the reversible processes. This results in the fact that vanishing entropy production is only necessary, but not sufficient for equilibrium.

Consequently, supplementary equilibrium conditions beyond the vanishing entropy production are required for equilibrium: that are vanishing entropy flux and vanishing time-space derivatives of the thermodynamic quantities. This results in the well known fact that the 4-temperature is a Killing vector in equilibrium for all materials.

Although not all results of this paper are really new, the method of deriving them is strict and does not depend on an ansatz of the entropy 4-vector. Beyond that, the used procedure can be easily extended to spin systems out of local equilibrium and to systems in General Relativity taking Einstein's field equations into account.

\section{References}

[1] C. Eckart: Thermodynamics of irreversible proceses III, Phys. Rev. 58 (1940) 919

[2] H. Stephani: General Relativity. An introduction to the theory of the gravitational field, Cambridge University Press, Cambridge 1982, 2nd edition 1990

[3] T. Chrobok, H.-H. v. Borzeszkowski: Thermodynamical equilibrium and spacetime geometry, Gen Relativ Gravit 38 (2006) 397-415

[4] W. Muschik, H.-H. v. Borzeszkowski: Entropy identity and materialindependent equilibrium conditions in relativistic thermodynamics, arXiv:0804.2659v1 [gr-qc] 16 Apr 2008 
[5] W. Muschik, H.-H. v. Borzeszkowski: Entropy identity and equilibrium conditions in relativistic thermodynamics. Gen Relativ Gravit 41 (2009 $1285-1304$

[6] W. Muschik, C. Papenfuss, H. Ehrentraut: A Sketch of Continuum Thermodynamics. J. Non-Newtonian Fluid Mech. 96 (2001) 255-290, Sect.3.4.

[7] W. Muschik, H.-H. v. Borzeszkowski: Exploitation of the Dissipation Inequality in General Relativistic Continuum Thermodynamics. Arch. Appl. Mech. 84 (2014) 1517-1531

[8] G. Neugebauer: Relativistische Thermodynamik, Vieweg Braunschweig (1980) ISBN 3-528-06863-9 